\title{
A participação do conselho de saúde mental na política da rede de atenção
}

\section{psicossocial}

\author{
The participation of the mental health council in the policy of the psychosocial care network \\ La participación del consejo de salud mental em la política de la red de atención psicosocial
}

Recebido: 17/08/2021 | Revisado: 22/08/2021 | Aceito: 25/08/2021 | Publicado: 27/08/2021

Vanessa Pinho

ORCID: https://orcid.org/0000-0002-4769-9117 Universidade Estadual do Ceará, Brasil E-mail: vanessaapinho14@gmail.com

Maria Salete Bessa Jorge

ORCID: https://orcid.org/0000-0001-6461-3015 Universidade Estadual do Ceará, Brasil E-mail: maria.salete.jorge@gmail.com

Dina Mara Formiga da Silva

ORCID: https://orcid.org/0000-0002-5387-7194 Universidade Estadual do Ceará, Brasil E-mail: dinamara_silva@hotmail.com

Débora Brenda Carneiro de Souza

ORCID: https://orcid.org/0000-0002-8503-4052 Universidade Estadual do Ceará, Brasil E-mail: deborabrenda@outlook.com

Cristiane Sales Leitão

ORCID: https://orcid.org/0000-0002-8412-0629 Assembleia Legislativa do Ceará, Brasil E-mail: cristianesalesleitao@gmail.com

Paulo Barroso

ORCID: https://orcid.org/0000-0002-4902-1940 Assembleia Legislativa do Ceará, Brasil E-mail: psi.pbarrodo@gmail.com

Lídia Lourinho

ORCID: https://orcid.org/0000-0002-5883-9007 Assembleia Legislativa do Ceará, Brasil E-mail: lidialourinho@ hotmail.com

\begin{abstract}
Resumo
Objetivo: Esse estudo buscou compreender a participação do Conselho de Saúde Mental na política da Rede de Atenção Psicossocial segundo a visão dos profissionais da saúde. Método: Trata-se de uma Revisão Integrativa que permite a busca e a análise crítica da produção científica relacionadas ao tema de investigação, onde foi seguido os seis passos de Mendes, Silveira e Galvão e a estratégia PCC (P=População, C=Conceito, C=Contexto). Resultados e discussões: Entre os artigos selecionados, os principais resultados obtidos dizem respeito a dois principais pontos: (1) lacuna entre política e prática; e (2) o desconhecimento/despreparo dos profissionais no âmbito da saúde mental. E para uma discussão mais completa, foi abordado um terceiro ponto: (3) o impacto da pandemia nos serviços de saúde mental. Considerações finais: Apesar de a maioria dos estudos se enquadrarem de acordo com as diretrizes da Política Nacional de Saúde Mental, muitas estratégias ainda carregam raízes do modelo biomédico.
\end{abstract}

Palavras-chave: Política de saúde mental; Atenção psicossocial.

\begin{abstract}
Objective: This study sought to understand the participation of the Mental Health Council in the policy of the Psychosocial Care Network according to the view of health professionals. Method: This is an Integrative Review that allows the search and critical analysis of scientific production related to the research theme, where the six steps of Mendes, Silveira and Galvão and the PCC strategy ( $\mathrm{P}=$ Population, $\mathrm{C}=$ Concept, $\mathrm{C}=\mathrm{Context}$ ) were followed. Results and discussions: Among the selected articles, the main results obtained relate to two main points: (1) gap between policy and practice; and (2) the lack of knowledge/unpreparedness of professionals in the mental health field. And for a more complete discussion, a third point was addressed: (3) the impact of the pandemic on mental health services. Final considerations: Although most studies fit according to the guidelines of the National Mental Health Policy, many strategies still bear roots of the biomedical model.
\end{abstract}

Keywords: Mental health policy; Psychosocial care. 


\begin{abstract}
Resumen
Objetivo: Este estudio buscó comprender la participación del Consejo de Salud Mental en la política de la Red de Atención Psicosocial de acuerdo con la visión de los profesionales de la salud. Método: Se trata de una Revisión Integrativa que permite la búsqueda y análisis crítico de la producción científica relacionada con el tema de investigación, donde se siguieron los seis pasos de Mendes, Silveira y Galvão y la estrategia pcc ( $\mathrm{P}=\mathrm{Población}$, $\mathrm{C}=$ Concepto, $\mathrm{C}=$ Contexto). Resultados y discusiones: Entre los artículos seleccionados, los principales resultados obtenidos se refieren a dos puntos principales: (1) brecha entre la política y la práctica; y (2) la falta de conocimiento/falta de preparación de los profesionales en el campo de la salud mental. Y para una discusión más completa, se abordó un tercer punto: (3) el impacto de la pandemia en los servicios de salud mental. Consideraciones finales: Aunque la mayoría de los estudios se ajustan a las directrices de la Política Nacional de Salud Mental, muchas estrategias todavía tienen raíces del modelo biomédico.
\end{abstract}

Palabras clave: Política de salud mental; Atención psicosocial.

\title{
1. Introdução
}

A Política Nacional de Saúde Mental (PNSM) ganhou força após anos de lutas e mobilizações populares que culminaram com a Reforma Psiquiátrica, embasada pela Lei $\mathrm{n}^{\circ} 10.216$, que dispõe sobre a proteção e os direitos das pessoas portadoras de transtornos mentais e redireciona o modelo assistencial em saúde mental (Ministério da Saúde, 2001). O que representou um grande avanço da saúde, redirecionando o cuidado à comunidade, assegurando direitos humanos àqueles antes esquecidos, excluídos e oprimidos.

Aliado a esse processo de redirecionamento do modelo assistencial em saúde mental, com a criação de serviços substitutivos ao hospital psiquiátrico tem-se a implementação da Rede de Atenção à Saúde (RAS) pelo Decreto 7.508/11, que é regulamentado pela Lei 8.080/1990, que dispõe sobre a organização do Sistema Único de Saúde (SUS), o planejamento da saúde, a assistência à saúde e a articulação Inter federativa, e dá outras providências (Ministério da Saúde, 2011).

Por conseguinte, houve a implementação Rede de Atenção Psicossocial (RAPS), instituída e regulamentada pela Portaria Ministerial 3.088/2011, com a finalidade de criar, ampliar e articular os pontos de atenção à saúde para pessoas com sofrimento ou transtorno mental e com necessidades decorrentes do uso de crack, álcool e outras drogas, no âmbito do SUS (Ministério da Saúde, 2011).

A Comissão Intersetorial de Saúde Mental (CISM) foi instituída em 1999 para acompanhar a Política Nacional de Saúde Mental, fornecendo subsídios ao Conselho Nacional de Saúde na área e garantindo o fortalecimento da Rede de Atenção Psicossocial (RAP). As ações da CISM buscam garantir a continuidade do Modelo de Atenção em Saúde Mental (desinstitucionalização) e da Reforma Psiquiátrica (Ministério da Saúde, 1999).

Já em dezembro de 2017, a Comissão Intergestora Tripartite (CIT) do Sistema Único de Saúde (SUS) se reuniu e foi aprovada, sem nenhum tipo de discussão, a resolução n. 32, a qual introduziu, na Rede de Atenção Psicossocial (RAPS), os ambulatórios de saúde mental e os leitos em hospitais psiquiátricos (HPs) (Ministério da Saúde, 2017). O que representa um retrocesso na luta por uma política antimanicomial, estimulando os proprietários de hospitais psiquiátricos a manterem esses serviços abertos, pois aumenta o incentivo financeiro a esse segmento (Souza \& Jorge, 2019).

Na prática, há uma ambivalência entre a legislação de saúde mental atual no país e o que os profissionais presenciam em seu dia a dia de assistência (Fernandes et al. 2020). O cuidado em saúde mental ainda enfrenta muitas barreiras que comprometem sua solidificação, como a instável articulação entre os equipamentos de saúde e sociais, a concretização da RP nas práticas dos serviços e dos profissionais, as dificuldades de alguns serviços em atender as demandas de saúde mental, entre outros (Nóbrega et al. 2017).

A fragilidade na formação de alguns profissionais que atuam nesse nível de atenção limita a qualidade dos atendimentos, pois muitas vezes não foram capacitados para trabalhar com essa demanda e acabam prestando uma assistência de baixa qualidade (Oliveira et al. 2020). 
Nota-se que ainda há muitos entraves para a implementação em sua totalidade nas políticas de saúde nas RAPS. Então, como ocorre a participação do conselho de saúde mental na política da Rede de Atenção Psicossocial sob a ótica dos profissionais da saúde? Diante ao exposto, o presente estudo tem como objetivo compreender a participação do Conselho de Saúde Mental na política da Rede de Atenção Psicossocial segundo a visão dos profissionais da saúde.

\section{Metodologia}

O estudo trata-se de uma Revisão Integrativa, o qual possibilita a busca e a análise crítica da produção científica, com o propósito de apresentar uma síntese das evidências relacionadas ao tema de investigação, o que permite verificar as possibilidades de desenvolver intervenções na área da saúde (Galvão \&Pereira, 2014).

Para a realização do estudo foi utilizados os seis passos estabelecidos por Mendes; Silveira \& Galvão (2008), 1) definição do tema e elaboração da pergunta de pesquisa; 2) critérios de elegibilidade, inclusão e exclusão dos estudos; 3) levantamento dos estudos selecionados em formato de tabelas, considerando todas as características em comum; 4) categorização e análise crítica dos achados, identificando diferenças e conflitos; 5) interpretação dos resultados e 6) apresentação dos resultados, incluindo análise crítica dos achados e síntese da revisão (Souza et al. 2010).

Para definir a pergunta norteadora foi utilizou-se a pergunta PCC, a estratégia PICO adaptada (Peters et al. 2015). Na adaptação, tais mnemônicos significam P- população, C- conceito e C- contexto/desfecho. Assim, a estratégia foi formulada da seguinte maneira: P- profissionais da saúde; C- políticas de saúde mental; C- Rede de Atenção Psicossocial.

Desta forma, estabeleceu-se, a seguinte questão: como ocorre a participação do conselho de saúde mental na política da rede de atenção psicossocial sob a ótica dos profissionais da saúde?

Após a identificação dos Descritores em Ciências da Saúde (DeCS), uma estratégia de busca detalhada, e individual, foi aplicada em cada base de dados: Pubmed, Biblioteca Virtual em Saúde (BVS), Web of Science. Utilizou-se três descritores extraídos do DeCS "pessoal de saúde", "política de saúde", "serviços de saúde mental" e duas palavras-chave "política de saúde mental", "atenção psicossocial", as quais reportam significativamente ao objeto de estudo.

E para a busca complementar utilizou-se da literatura cinza via Google Scholar, sites de órgãos governamentais e serviços de saúde para levantamento de manuais sobre Saúde Mental. A literatura cinza é definida por Cunha \& Cavalcante (2008, p.230) como o "conjunto de documentos impressos ou multicopiados que não são nem editados, nem difundidos comercialmente. Circulam, geralmente, em congressos, reuniões e centros de pesquisa [...]".

Durante a estratégia de busca, foram elencados como critérios de inclusão: artigos completos disponíveis integralmente nas bases de dados elencadas nos idiomas português, inglês e espanhol e selecionados, artigos referentes aos últimos cinco anos, informações complementares utilizando-se sites de órgãos governamentais e serviços de saúde; publicações que atendessem a pergunta norteadora. Foram excluídas publicações que preenchiam pelo menos um dos seguintes critérios: (1) revisões; (2) cartas ao editor, (3) opinião pessoal de autores; (4) resumo de encontros; (5) vídeos; (6) ausência de dados relacionados ao objeto e estudo. 
Quadro 1 - Equações de buscas nas bases de dados.

\begin{tabular}{|c|c|}
\hline BASE & ESTRATÉGIA DE BUSCA \\
\hline PubMed & ((Health Personnel) AND (Policy Health)) AND (Mental Health Service) \\
\hline BVS & "pessoal de saúde" AND "política de saúde” AND "serviço de saúde mental” \\
\hline Web of Science & (Health Personnel) AND (Policy Health) AND (Mental Health Service) \\
\hline Google Scholar & "política de saúde mental” AND "atenção psicossocial” \\
\hline
\end{tabular}

Fonte: Autores (2021).

A combinação dos termos foi selecionada e adaptada para cada base de dados (Quadro 1). O software Endnotweb foi selecionado para o manejo de todas as referências e a remoção dos artigos duplicados. Para a análise dos dados, foi utilizada a Análise de Conteúdo de Bardin (2011), estabelecendo assim categorias.

\section{Resultados}

Nesta pesquisa, foram analisados 11 artigos científicos que atenderam aos critérios de inclusão estabelecidos previamente. Para facilitar a análise e apresentação dos resultados, elaborou-se o (Quadro 1) com dados sobre o título, autor, idioma, periódico, tipo de produção e principais resultados de cada estudo em relação à temática.

Dos onze artigos selecionados, três não são nacionais, estando disponível na íntegra apenas na língua inglesa. Os demais artigos estavam disponíveis nos três idiomas incluídos no estudo: português, inglês e espanhol. Quanto aos aspectos metodológicos, observou-se predominância de estudos com abordagem qualitativa. Já em relação ao periódico das publicações, destaca-se a Revista Brasileira de Enfermagem ( $n=2)$. 
Figura 1 - Fluxograma da seleção das publicações para a revisão integrativa baseado no modelo PRISMA.

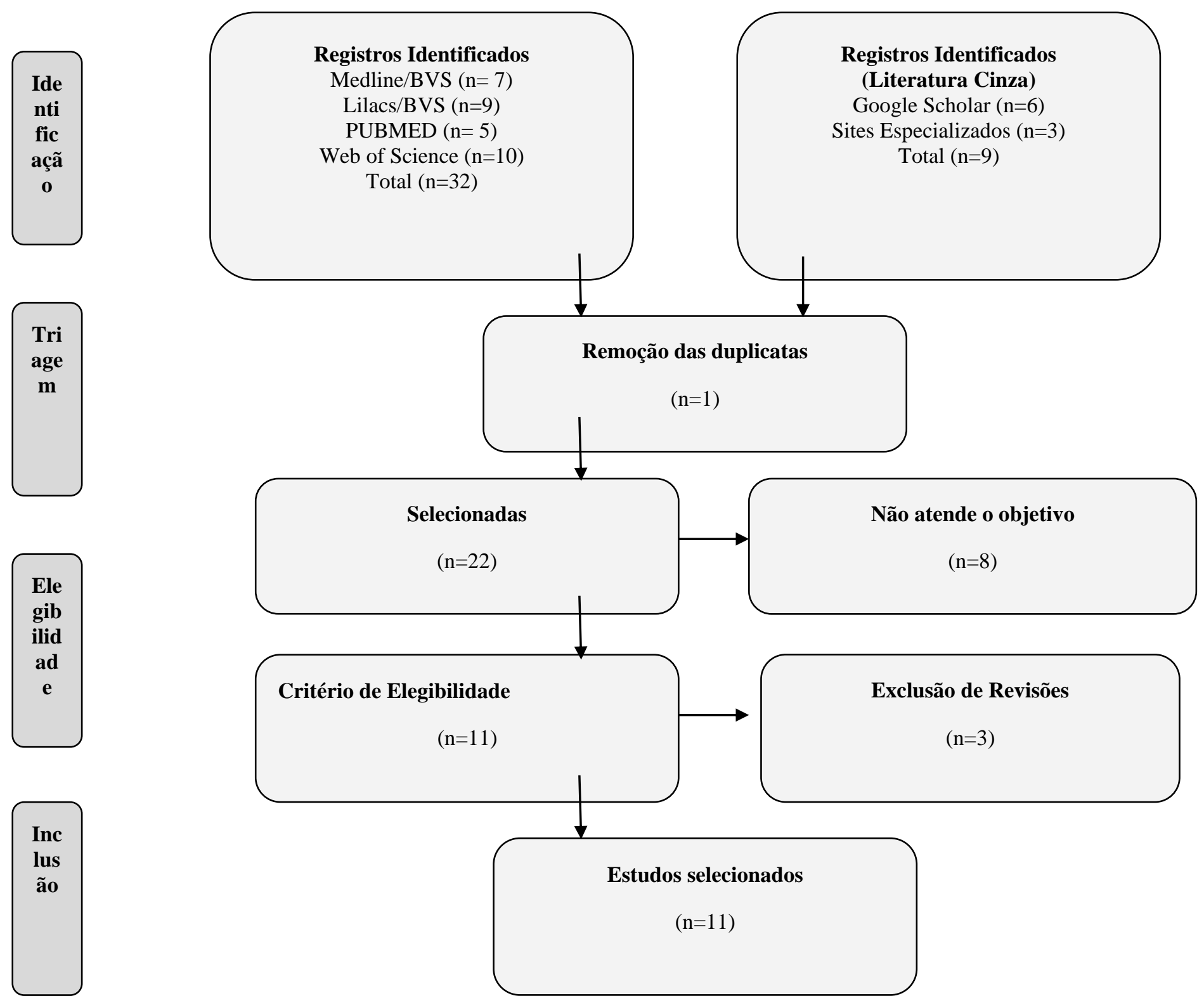

Fonte: Autores (2021).

As etapas deste processo foram descritas na forma de um fluxograma (Figura 1) adaptado do Reporting Items Systematic and Meta-Analyses (PRISMA) restando $(\mathrm{n}=10)$ para serem analisados e construída a matriz de síntese (Quadro 2) 
Quadro 2 - Caracterização dos estudos incluídos na Revisão Integrativa.

\begin{tabular}{|c|c|c|c|c|c|}
\hline Título & Autor & Idioma & Periódico & Tipo de publicação & \begin{tabular}{|c|} 
Principais resultados \\
\end{tabular} \\
\hline $\begin{array}{l}\text { Tecendo a Rede de } \\
\text { Atenção Psicossocial } \\
\text { Oeste do Município de } \\
\text { São Paulo. }\end{array}$ & $\begin{array}{l}\text { Nóbrega } \\
\text { MDPSS, } \\
\text { Domingos } \\
\text { AM, Silveira } \\
\text { ASA, Santos } \\
\quad \text { JCD }\end{array}$ & $\begin{array}{l}\text { Inglês / } \\
\text { Portuguê } \\
\quad \mathrm{s}\end{array}$ & $\begin{array}{l}\text { Rev. Bras. } \\
\text { Enferm. }\end{array}$ & $\begin{array}{c}\text { Pesquisa de abordagem } \\
\text { qualitativa. }\end{array}$ & $\begin{array}{c}\text { A rede está em processo de alinhamento com a política de saúde mental } \\
\text { e a estratégia de reabilitação psicossocial. No entanto, existem } \\
\text { fragilidades no processo de trabalho das equipes, relacionadas ao déficit } \\
\text { de recursos humanos, estruturais e de comunicação e à limitada } \\
\text { apropriação de suas diretrizes. }\end{array}$ \\
\hline $\begin{array}{l}\text { Conhecimento dos } \\
\text { Gestores sobre a Política } \\
\text { Nacional de Saúde } \\
\text { Mental. }\end{array}$ & $\begin{array}{l}\text { Fernandes RL, } \\
\text { Miranda FAN, } \\
\text { Oliveira KKD, } \\
\text { Rangel CT, } \\
\text { Costa } \\
\text { DARDS, } \\
\text { Moura IBL }\end{array}$ & $\begin{array}{l}\text { Inglês/ } \\
\text { Portuguê } \\
\quad \text { s/ } \\
\text { Espanhol }\end{array}$ & $\begin{array}{l}\text { Rev. Bras. } \\
\text { Enferm. }\end{array}$ & $\begin{array}{l}\text { Estudo descritivo, de natureza } \\
\text { qualitativa. }\end{array}$ & $\begin{array}{l}\text { Os resultados produziram as seguintes categorias: De volta à sociedade: } \\
\text { protagonismo e autonomia dos pacientes; Equipe interprofissional: } \\
\text { atribuições e atividades; Estruturação de uma rede de atenção } \\
\text { psicossocial; Desafios que afetam o serviço; Distância entre a política e } \\
\text { a prática. }\end{array}$ \\
\hline $\begin{array}{l}\text { O acolhimento na Rede } \\
\text { de Atenção Psicossocial: } \\
\text { estudo descritivo } \\
\text { exploratório. }\end{array}$ & $\begin{array}{l}\text { Oliveira AB, } \\
\text { Barbosa VFB, } \\
\text { Silva AC, } \\
\text { Santos ASC, } \\
\text { Santos } \\
\text { DCS. }\end{array}$ & $\begin{array}{l}\text { Inglês/Po } \\
\text { rtuguês/ } \\
\text { Espanhol } \\
\end{array}$ & $\begin{array}{l}\text { Revista Online } \\
\text { braz. j. nurs. }\end{array}$ & $\begin{array}{l}\text { Trata-se de um estudo } \\
\text { descritivo e exploratório, de } \\
\text { abordagem qualitativa. }\end{array}$ & $\begin{array}{l}\text { As práticas de acolhimento predominantes foram a escuta qualificada, } \\
\text { o estabelecimento de vínculo e o diálogo. Evidenciou-se consonância } \\
\text { com a Política Nacional de Humanização e com o modelo de atenção } \\
\text { psicossocial. }\end{array}$ \\
\hline $\begin{array}{l}\text { A supervisão clínico- } \\
\text { institucional como } \\
\text { dispositivo de } \\
\text { qualificação na Atenção } \\
\text { Psicossocial: uma } \\
\text { experiência de parceria } \\
\text { com a Universidade. }\end{array}$ & $\begin{array}{l}\text { Lima CH, } \\
\text { Sampaio } \\
\text { TCSM, } \\
\text { Cunha JS. }\end{array}$ & $\begin{array}{l}\text { Inglês/ } \\
\text { Portuguê } \\
\quad \text { S }\end{array}$ & $\begin{array}{l}\text { Physis: } \\
\text { Revista de } \\
\text { Saúde } \\
\text { Coletiva. }\end{array}$ & $\begin{array}{l}\text { Metodologia qualitativa de } \\
\text { construção do caso clínico. }\end{array}$ & $\begin{array}{l}\text { Destaca-se a psicanálise como marco teórico principal na análise } \\
\text { sobre a forma como o cotidiano dos usuários do CAPSII se situa no } \\
\text { horizonte da reforma psiquiátrica, sobre o papel da psiquiatria no CAPS } \\
\text { e na construção de um } \\
\text { saber alternativo à psiquiatria, que considere a relevância da teoria, da } \\
\text { posição subjetiva dos usuários e do saber dos profissionais. }\end{array}$ \\
\hline
\end{tabular}


Research, Society and Development, v. 10, n. 11, e177101119540, 2021

(CC BY 4.0) | ISSN 2525-3409 | DOI: http://dx.doi.org/10.33448/rsd-v10i11.19540

\begin{tabular}{|c|c|c|c|c|c|}
\hline $\begin{array}{l}\text { rmações para sistemas de } \\
\text { saúde mental: um } \\
\text { instrumento para } \\
\text { formulação de políticas e } \\
\text { qualidade de serviço do } \\
\text { sistema. }\end{array}$ & $\begin{array}{l}\text { Lora, A; } \\
\text { Lesage, A; } \\
\text { Pathare, S } \\
\text { Levav, I. }\end{array}$ & Inglês & $\begin{array}{l}\text { Epidemiol } \\
\text { Psychiatr Sci }\end{array}$ & $\begin{array}{l}\text { Publicações e pesquisas } \\
\text { relevantes foram consultadas, } \\
\text { incluindo estudos da OMS que } \\
\text { pretendem promover o uso de } \\
\text { sistemas de informação para } \\
\text { melhorar a atenção à saúde } \\
\text { mental em países de alta e } \\
\text { média baixa renda. }\end{array}$ & $\begin{array}{c}\text { Estudos têm mostrado que, uma vez que as informações tenham sido } \\
\text { coletadas por sistemas relevantes e analisadas por meio de esquemas de } \\
\text { indicadores, elas podem ter diversos usos. O monitoramento dos } \\
\text { serviços de saúde mental representa um primeiro passo no uso da } \\
\text { informação. }\end{array}$ \\
\hline $\begin{array}{l}\text { Como os cuidados de } \\
\text { saúde mental devem } \\
\text { mudar como } \\
\text { consequência da } \\
\text { pandemia Covid-19. }\end{array}$ & $\begin{array}{l}\text { Moreno, C; } \\
\text { Männikkö, M. } \\
\text { Phillips, M. R. } \\
\text { Uchida, H. } \\
\text { Vieta, E. } \\
\text { Vita, A. } \\
\text { Arango, C. }\end{array}$ & Inglês & $\begin{array}{l}\text { Lancet } \\
\text { Psychiatry }\end{array}$ & $\begin{array}{l}\text { Para este Documento de } \\
\text { Posição, um grupo } \\
\text { internacional de médicos, } \\
\text { especialistas em saúde mental } \\
\text { e usuários de serviços de } \\
\text { saúde mental se reuniram para } \\
\text { refletir sobre os desafios para } \\
\text { a saúde mental que o COVID- } \\
19 \text { apresenta. }\end{array}$ & $\begin{array}{c}\text { A interconexão do mundo tornou a sociedade vulnerável a esta } \\
\text { infecção, mas também fornece a infraestrutura para lidar com as falhas } \\
\text { anteriores do sistema, disseminando boas práticas que podem resultar } \\
\text { na prestação sustentada, eficiente e eqüitativa de serviços de saúde } \\
\text { mental. }\end{array}$ \\
\hline $\begin{array}{l}\text { Desarticulação da rede } \\
\text { psicossocial } \\
\text { comprometendo a } \\
\text { integralidade do } \\
\text { cuidado. }\end{array}$ & $\begin{array}{l}\text { Carvalho } \\
\text { MFAA, } \\
\text { Coelho EAC, } \\
\text { Oliveira JF, } \\
\text { Araújo RT, } \\
\text { Barros AR }\end{array}$ & $\begin{array}{l}\text { Inglês/Po } \\
\text { rtugues }\end{array}$ & $\begin{array}{l}\text { Rev. da Escola } \\
\text { de Enf. da } \\
\text { USP. }\end{array}$ & $\begin{array}{c}\text { Pesquisa de abordagem } \\
\text { qualitativa. }\end{array}$ & $\begin{array}{c}\text { Nove profissionais } \\
\text { participaram do estudo. Todos relataram fazer parte de um serviço } \\
\text { desarticulado }\end{array}$ \\
\hline $\begin{array}{l}\text { Competição Entre } \\
\text { Organizações de Saúde } \\
\text { Mental: Ambientes } \\
\text { motivadores e respostas } \\
\quad \text { estratégicas. }\end{array}$ & $\begin{array}{l}\text { Bunger, AC, } \\
\text { Choi, MS, } \\
\text { MacDowell, } \\
\text { Gregoire, T. }\end{array}$ & Inglês. & $\begin{array}{l}\text { Administratio } \\
\mathrm{n} \text { and Policy } \\
\text { in Mental } \\
\text { Health and } \\
\text { Mental Health } \\
\text { Services } \\
\text { Research. }\end{array}$ & $\begin{array}{l}\text { Pesquisa de abordagem } \\
\text { qualitativa. }\end{array}$ & $\begin{array}{l}\text { A análise revelou duas grandes correntes temáticas sobre a competição } \\
\text { entre organizações regionais de saúde mental. Um fluxo surgiu em } \\
\text { torno da competição intensiva por pessoal qualificado, e um segundo } \\
\text { focado na competição limitada / estável por clientes e recursos } \\
\text { financeiros. Os motivadores e as respostas a esses diferentes tipos de } \\
\text { competição foram distintos. }\end{array}$ \\
\hline
\end{tabular}

Fonte: Autores (2021). 
Entre os artigos selecionados, os principais resultados obtidos dizem respeito a dois principais pontos: (1) lacuna entre política e prática; e (2) o desconhecimento/despreparo dos profissionais no âmbito da saúde mental. Por fim, para uma discussão mais completa, será abordado um terceiro ponto adicional: (3) o impacto da pandemia nos serviços de saúde mental

\section{Discussões}

\subsection{Política: entre teoria e prática}

A centralidade no usuário foi situada como diretamente proporcional à descentralização da psiquiatria, implicando a modificação na relação entre a equipe responsável por seu acolhimento e abordagem e o sofrimento psíquico que o atravessa (Lima; Sampaio \& Cunha, 2019). Sobre isso, pode-se afirmar:

Reconhece-se que o processo da Reforma Psiquiátrica brasileira, sua política e o modelo proposto de atenção psicossocial passam por um processo de metamorfose em que coexistem práticas arraigadas e um modelo hegemônico herdado da psiquiatria clássica que reforça, de um lado, o modelo biomédico e medicalizante, de outro, o redesenho de um campo novo, onde diversos atores interagem para desconstruir o modelo herdado e construir um modelo compartilhado de atenção à saúde mental (Fernandes et al. 2020, p.5).

Pode-se perceber que apesar de um histórico de reformas políticas, Bunger et al. (2021) aponta que os sistemas de saúde mental ainda sofrem problemas persistentes de acessibilidade, coordenação e qualidade nos Estados Unidos e no mundo. O quadro atual da política de saúde mental no Brasil vem promovendo a revalorização do modelo hospitalocêntrico, tanto pela inserção dos hospitais psiquiátricos na RAPS como pelo aumento de investimento, desde 2017, para os hospitais psiquiátricos (Lima; Sampaio \& Cunha, 2019).

O financiamento em saúde, e especificamente, em saúde mental são baixos e pouco eficazes, Trapé \& Campos (2017) trouxe que a Organização Mundial da Saúde investe em torno de 5\% do orçamento da saúde em saúde mental, em decorrência da sua prevalência e do impacto assistencial. Já o Governo Federal direciona somente 2,3\% do orçamento total da saúde em ações específicas de saúde mental. Esse valor aumenta timidamente se considerarmos a saúde mental na atenção primária e os Núcleos de Apoio à Saúde da Família (NASF).

Destacou-se também que a desarticulação entre a RAPS gera uma centralização nos Centros de Atenção Psicossocial (CAPS), pois seus usuários permanecem por tempo indeterminado já que, segundo os estudos, falta suporte nos serviços para dar continuidade ao tratamento (Carvalho et al. 2017). A saúde mental ainda está presa a uma tradição ultrapassada e um déficit de treinamento, ficando atrás no sistema geral de saúde na coleta e análise de informações padrão (Lora et al. 2017). Muitos muros concretos e simbólicos de exclusão da loucura precisam ser quebrados para que as pessoas com transtorno mental possam viver com mais dignidade (Jafelice; Ziliotto \& Marcolan, 2020).

\subsection{Desconhecimento/despreparo dos profissionais}

Após a leitura dos estudos, notou-se que um ponto em comum entre eles diz respeito ao pouco conhecimento sobre os princípios e diretrizes da RAPS. Muitos profissionais não receberam orientação e não compreendem o novo processo, algo justificado por ser uma política recente e desafiadora. A rotatividade de profissionais associada à elevada demanda de usuários acarreta muitas dificuldades na dinâmica da assistência de forma mais ampliada. (Nóbrega et al. 2017). Em seu estudo com 123 profissionais da RAPS da região oeste de São Paulo, Nóbrega et al. (2017), aponta que: 
Há necessidade de transformação na formação acadêmica e continuada dessas equipes, e aproximação da APS com os serviços especializados para enriquecer o arcabouço que sustenta ações de saúde mental nesse cenário, e conseguir dar conta das demandas do território. Partindo desse princípio, o processo de formação e educação permanente deve estar em concordância com os princípios da PNSM vigente e com as diretrizes para o funcionamento da RAPS (p.1021).

No que diz respeita à gestão do cuidado foi percebido alguns problemas estruturais como espaços físicos ineficientes, falta de educação permanente, baixa articulação da Rede de Atenção Psicossocial e ainda a presença do estigma criando obstáculo no processo de ressocialização dos usuários (Fernandes et al. 2020).

De modo geral, os profissionais não têm um padrão específico de acolhimento a ser seguido na assistência a pessoas com adoecimento mental. Apesar disso, notou-se que suas condutas são baseadas na humanização, e muitos utilizam as ferramentas de escuta qualificada e diálogo, primordiais para conhecer a trajetória do usuário e com isso estabelecer um vínculo com ele (Oliveira et al. 2020).

\section{3 o impacto da pandemia de covid-19 aos serviços de saúde mental}

A pandemia de covid aconteceu de forma repentina e inesperada, trazendo grande impactos para os serviços de saúde no mundo inteiro, inclusive no âmbito da saúde mental, por isso, um dos artigos selecionados trata somente em relação a essa temática. Moreno et al. (2020) cita em seu artigo vários estudos que diz:

As medidas para promover o distanciamento físico incluem a redução do número de consultas ambulatoriais, a adoção de protocolos de triagem que recomendam o tratamento apenas de questões urgentes, e a reestruturação do número de casos para minimizar o contato entre os pacientes. Psicoterapia de grupo e reuniões de apoio de pares foram reduzidas, canceladas ou movidas online. As unidades psiquiátricas de internação têm estimulado o distanciamento físico por meio do uso de quartos de isolamento, diminuindo o número total de leitos disponíveis, restringindo mais a admissão e reduzindo o tempo de internação (Moreno et al. 2020, p. 815).

Ainda não se pode prever totalmente os efeitos da pandemia, mas é notável que a doença mexeu com o psicológico tanto da população como das pessoas em adoecimento mental e trabalhadores da linha de frente. Apesar da diversidade nos sistemas políticos, sociais e de saúde, os serviços de saúde mental adotaram respostas agudas focando no controle de infecção em vários países, como ajustes nas políticas de acesso, cobertura de seguro, leis de privacidade e acesso a medicamentos controlados (Moreno et al. 2020).

\section{Considerações Finais}

Diante do exposto, pode-se concluir que muitas estratégias ainda carregam raízes do modelo biomédico, apesar de a maioria dos estudos se enquadrarem de acordo com as diretrizes da Política Nacional de Saúde Mental, embasado na Lei 10.216. De forma geral, percebeu-se dificuldade em achar pesquisas que falassem diretamente sobre o Conselho de Saúde Mental (CISM).

Uma série de barreiras ainda afetam a gestão do cuidado em saúde mental, por isso, é recomendado que é importante haver uma maior conexão entre os serviços e os profissionais atuantes nele, em relação a estrutura física, recursos materiais e humanos, capacitações e educação permanente. A demanda de trabalho em saúde mental exige uma equipe atualizada a fim de romper de vez com práticas que vão contra os princípios da Reforma Psiquiátrica. O despreparo dos profissionais em saúde mental tanto em relação à assistência quanto em relação ao conhecimento político compromete o cuidado integral. 
Research, Society and Development, v. 10, n. 11, e177101119540, 2021

(CC BY 4.0) | ISSN 2525-3409 | DOI: http://dx.doi.org/10.33448/rsd-v10i11.19540

Por fim, vale ressaltar o impacto da pandemia de Covid-19 nos serviços de saúde e nos serviços de saúde mental, trazendo diversos impactos negativos sobre o estado psicológico da sociedade, dos profissionais e das pessoas em sofrimento mental. Este estudo foi relevante pois apesar de existir um vasto campo de pesquisa sobre saúde mental, poucas focam no âmbito político e no conselho de saúde mental, principalmente no que diz respeito às dificuldades e às barreiras ainda existentes, ainda mais no contexto atual.

Ante ao exposto, sugere-se mais estudos que foquem na participação do Conselho de Saúde Mental, visando a teoria e sua eficácia na prática cotidiana, já que os resultados mostram o despreparo de grande parte dos profissionais em saúde mental tanto em relação à assistência quanto em relação ao conhecimento político, comprometendo o cuidado integral.

\section{Referências}

Brasil. (2001). Lei 10.216, de 06 de abril de 2001. Dispõe sobre a proteção e os direitos das pessoas portadoras de transtornos mentais e redireciona o modelo assistencial em saúde mental. Ministério da Saúde. http://www.planalto.gov.br/ccivil_03/leis/leis_2001/110216.htm.

Brasil. (2011). Decreto $n^{\circ} 7.508$, de 28 de junho de 2011. Regulamenta a Lei $n^{\circ} 8.080$, de 19 de setembro de 1990 , para dispor sobre a organização do Sistema Único de Saúde - SUS, o planejamento da saúde, a assistência à saúde e a articulação interfederativa, e dá outras providências. Ministério da Saúde. http://www.planalto.gov.br/ccivil_03/_ato2011-2014/2011/decreto/d7508.htm.

Brasil. (2017). Comissão Intergestores Tripartite. Resolução n. 32, de 14 de dezembro de 2017, Ministério da Saúde. https://www.gov.br/cidadania/ptbr/acesso-a-informacao/legislacao/resolucao-cit-no-32-2017-de-17-de-dezembro-de-2017.

Brasil. (1999). Comissão Intersetorial de Saúde Mental. Conselho Nacional de Saúde. Ministério da Saúde. http://conselho.saude.gov.br/comissoes-cns/cism

Brasil. (2011). Portaria $n^{o}$. 3088, de 23 de dezembro de 2011. Institui a Rede de Atenção Psicossocial para pessoas com sofrimento ou transtorno mental e com necessidades decorrentes do uso de crack, álcool e outras drogas, no âmbito do Sistema Único de Saúde. Ministério da Saúde. https://bvsms.saude.gov.br/bvs/saudelegis/gm/2011/prt3088_23_12_2011_rep.html.

Bunger, A. C., Choi, M. S. \& Macdowell, H. et al. (2021). Competition Among Mental Health Organizations: Environmental Drivers and Strategic Responses. Adm Policy Ment Health 48, 393-407. https://doi.org/10.1007/s10488-020-01079-2.

Carvalho M. F. A. A., Coelho E. A. C., Oliveira J. F., Araujo R. T. \& Barros A. R. (2017). Uncoordinated psychosocial network compromising the integrality of care. Rev Esc Enferm USP. http://dx.doi.org/10.1590/S1980-220X2016040703295.

Cunha, M. B. \& da Cavalcanti, C. R. O. (2008). Dicionário de Biblioteconomia e Arquivologia. Brasília: Briquet de Lemos, 2. https://repositorio.unb.br/handle/10482/34113.

EndNote Web (2021). [place unknown]; https://access.clarivate.com/login?app=endnote

Fernandes R. L., Miranda F. A. N., Oliveira K. K. D., Rangel, C. T., Costa D. A. R. S. \& Moura I. B. L. (2020). Knowledge of managers on the National Mental Health Policy. Rev Bras Enferm; 73(1): e20180198. https://doi.org/10.1590/0034-7167-2018-0198.

Galvao, T. F. \& Pereira, M. G. (2014). Revisões sistemáticas da literatura: passos para sua elaboração. Epidemiol. Serv. Saúde, Brasília, v. 23, n. 1, p. 183-184. http://scielo.iec.gov.br/scielo.php?script=sci_arttext\&pid=S1679-49742014000100018.

Jafelice, G. T., Ziliotto, G. C. \& Marcolan, J. F. (2020). Concepção dos profissionais do centro de atenção psicossocial sobre políticas públicas de saúde mental. Pesquisa, Sociedade e Desenvolvimento, [S. l.] 9(5), e71953100. https://doi.org/10.33448/rsd-v9i5.3100.

Lima, C. H; Sampaio, T. C. S. M. \& Cunha, J. S. (2019). A supervisão clínico-institucional como dispositivo de qualificação na Atenção Psicossocial: uma experiência de parceria com a Universidade. Physis: Revista de Saúde Coletiva, 29(3), e290314. https://doi.org/10.1590/S0103-73312019290314.

Lora, A,, Lesage A., Pathare S., \& Levav I.(2017). Information for mental health systems: an instrument for policy-making and system service quality. Epidemiol Psychiatr Sci; 26(4):383-394. https://doi.org/10.1017/S2045796016000743.

Mendes K. D. S., Silveira, R. C. C. P. \& Galvão, C. M. (2008). Revisão integrativa: método de pesquisa para a incorporação de evidências na saúde e na enfermagem. Texto Contexto Enferm, 17(4): 758-64. https://doi.org/10.1590/S0104-07072008000400018.

Moreno, C., et al. (2020). How mental health care should change as a consequence of the Covid-19 pandemic. Lancet Psychiatry ;7(9):813-824. https://doi.org/10.1016/S2215-0366(20)30307-2.

Nobrega, M. P. S. S., Domingos, A. M., Silveira, A. S. A. \& Santos J. C. (2017). Weaving the West Psychosocial Care Network of the municipality of São Paulo. Rev Bras Enferm ;70(5):965-72. [Thematic Edition "Good practices and fundamentals of Nursing work in the construction of a democratic society"] http://dx.doi.org/10.1590/0034-7167-2016-0566.

Oliveira, A. B et al. (2020). O acolhimento na rede de atenção psicossocial: estudo descritivo-exploratório. Online braz. j. nurs. (Online); 19(2) https://pesquisa.bvsalud.org/portal/resource/pt/biblio-112205 . 
Research, Society and Development, v. 10, n. 11, e177101119540, 2021 (CC BY 4.0) | ISSN 2525-3409 | DOI: http://dx.doi.org/10.33448/rsd-v10i11.19540

Peters, M. D et al. (2015). Guidance for conducting systematic scoping reviews. Int J Evid Based Healthc;13(3):141-6. https://doi.org/10.1097 / XEB.0000000000000050

Sousa, F. S. P. \& Jorge, M. S. B. (2019). O retorno da centralidade do hospital psiquiátrico: retrocessos recentes na política de saúde mental. Trab. Educ. Saúde, 17(1):e0017201. https://doi.org/10.1590/1981-7746-sol00172.

Trape T. L. \& Onocko-Campos R. (2017). Modelo de atenção à saúde mental do Brasil: análise do financiamento, governança e mecanismos de avaliação. Rev Saude Publica. 51:19. https://doi.org/10.1590/S1518-8787.2017051006059.

Tricco A. C., Lillie E., Zarin W., O’Brien K. K., Colquhoun H. \& Levac D, et al. (2018). PRISMA Extension for Scoping Reviews (PRISMA-ScR): Checklist and Explanation. Ann Intern Med 4; 169(7):467-73. https://www.acpjournals.org/doi/abs/10.7326/M18-0850. 